\title{
Philosophiques
}

Jean-Louis Allard. L'éducation à la liberté ou la philosophie de l'éducation de Jacques Maritain. Ottawa et Grenoble, Les Éditions de l'Université d'Ottawa et les Presses universitaires de Grenoble, 1978, 152 p.

\section{André Vidricaire}

Volume 8, numéro 1, avril 1981

URI : https://id.erudit.org/iderudit/203162ar

DOI : https://doi.org/10.7202/203162ar

Aller au sommaire du numéro

Éditeur(s)

Société de philosophie du Québec

ISSN

0316-2923 (imprimé)

1492-1391 (numérique)

Découvrir la revue

Citer ce compte rendu

Vidricaire, A. (1981). Compte rendu de [Jean-Louis Allard. L'éducation à la liberté ou la philosophie de l'éducation de Jacques Maritain. Ottawa et

Grenoble, Les Éditions de l'Université d'Ottawa et les Presses universitaires de Grenoble, 1978, 152 p.] Philosophiques, 8(1), 206-211.

https://doi.org/10.7202/203162ar d'utilisation que vous pouvez consulter en ligne. 
Jean-Louis ALLARD. L'éducation à la liberté ou la philosophie de l'éducation de Jacques Maritain. Ottawa et Grenoble, Les Éditions de l'Université d'Ottawa et les Presses universitaires de Grenoble, $1978,152 \mathrm{p}$.

Voici un livre sur Maritain, qui pourrait et devrait normalement traiter de questions et de problèmes qui assaillent non seulement la culture d'ici, mais encore diverses disciplines et cette philosophie toujours en train de se faire. En un mot, Maritain peut stimuler la passion et la lucidité. Un livre sur ce philosophe peut être une entreprise périlleuse, parce que située au coeur de débats viscéraux et urgents. Mais où est le danger?

C'est bien là le problème! Avec Allard, il n'y a pas d'enjeu explicite. J'ai beau chercher: dans la préface, dans l'introduction, dans le résumé du chapitre VI, j'ignore pourquoi Allard a fait un livre sur la philosophie de l'éducation de Maritain. L'auteur a beau répéter qu'il dégage les articulations fondamentales de la pensée éducative de Maritain dans leur continuité avec leurs fondements philosophiques (p. 16, 24,129,139), ça ne me dit pas pourquoi il s'intéresse à cela plutôt qu’à la pensée religieuse des évêques du Québec. Je m’explique.

Si le propos d'Allard consiste à montrer que la pédagogie du philosophe repose sur une conception philosophique, de multiples questions surgissent qui assaillent notamment des historiens de la philosophie comme Alquié, Rodis-Lewis, Guéroult, Pérelman, Gouhier, etc. Par exemple, sur quoi repose l'«unité» d'une doctrine philosophique? Comment un philosophe qui traite de 
réalités aussi hétérogènes que l'éducation, la politique, la poésie, la connaissance, parvient-il à une vision cohérente? Si on refuse l'idée que l'unité d'une pensée repose sur une logique et une architectonique, comme semble le dire Maritain, sur quoi faudra-t-il s'appuyer? Sur une expérience de l'être, sur une démarche de la raison, sur une intuition? Mais encore là, ces notions sont loin d'être évidentes: juxtaposez et comparez les idées d'Alquié, Bergson, Gilson et même de Maritain au sujet de l'intuition! Néanmoins, malgré l'imprécision, vous verrez que chacun d'eux comme historien cherche à cerner l'intuition philosophique de Platon, de saint Thomas ou d'un autre. Et Allard procède de la même façon quand il écrit: «Nous avons lu . . . la plupart des oeuvres de Maritain afin d'en découvrir les intuitions fondamentales» (p. 129). Ce faisant, je ne dis pas que ces chercheurs émérites se trompent; je dis plutôt que, dans l'horizon d'une histoire des doctrines, d'une histoire des idées, d'une histoire de la philosophie, d'une histoire des philosophies, d'une histoire des visions du monde, d'une histoire des pensées et/ou des courants philosophiques, etc., la plupart de ceux qui étudient présentement le texte philosophique sont aux prises avec des questions très complexes étrangères à Allard. Dès lors, quand ce dernier veut montrer que la pédagogie de Maritain repose sur une conception philosophique de la personne et de la liberté, son propos ne consiste pas, comme Gueroult tente de le faire, à résoudre des problèmes d'histoire de la philosophie ni de la phiłosophie comme savoir, doctrine, système, intuition, vérité universelle. Allard ne veut pas non plus, à un autre niveau, établir si, entre la pédagogie et la philosophie de Maritain, existe une simple relation d'implication ou encore une relation de présupposition. Néanmoins, Allard écrit sans le démontrer que l'éducation, pour Maritain, consiste à conquérir une liberté d'autonomie et d'épanouissement à la fois dans l'ordre personnel et dans l'ordre social, et se fonde sur une conception thomiste et chrétienne de l'homme comme personne spirituelle. C'est pourquoi il conclut: "La pédagogie de Maritain nous semble être un reflet fidèle de sa conception de la philosophie» (p. 131). En l'absence de tout procédé explicite d'analyse qui permet d'aboutir à cette conclusion, je partage l'affirmation modalisée du «nous semble»... .

Mais comme je l'écrivais plus haut, ce n'était pas un des problèmes qui préoccupent actuellement ceux qui s'interrogent sur la constitution d'un texte, d'une doctrine, d'une pensée. Mais que voulait bien faire Allard qui traite, à mon avis, d'un sujet très utile dans la culture d'ici et de l'Occident? Analyser la conception éducative de Maritain? En établir la pertinence dans le cadre des débats éthiques et sociaux? Approfondir et thématiser certaines informations de R. Houde (cf. Relations, $\mathrm{n}^{\circ}$ 383-384; juin et juillet-août 1973) sur la présence de Maritain à Montréal à l'époque de la Deuxième Guerre? En un mot, où est l'urgence? où est l'enjeu? où est le péril? où est le problème? où est la question qui dépasse la seule énumération des idées de Maritain sur l'éducation morale, le rôle spécifique de l'école, l'école chrétienne, les objectifs et les contenus généraux des écoles secondaires et universitaires? Allard traite de tous ces sujets, mais ils ne sont pas thématisés; ils ne sont pas formulés sous la forme d'un problème à l'aide de concepts. Pourtant, l'occasion était belle d'interroger, par exemple, la constitution de ce modèle pédagogique: ce qui aurait évité 
les rapprochements confus et piégés de Maritain avec Freire et Neill. En effet, comme il s'agit d'un "savoir", sa formation et sa constitution renvoient à des notions de "courant", «approche» ou de «famille» qui définit une "centration", à des notions de «situations d'enseignement" et d'éléments génériques qui les définissent, dont par exemple «la direction», «les clients et leurs attentes", etc. Après Joyce et Weil, au Québec Ginette Lépine, Pierre Angers, Yves Bertrand et des services du MEQ traitent maintenant de ces questions épistémologico-culturelles. Mais si Allard préférait s'en tenir à ce qu'on a coutume d'appeler «les grandes questions de la philosophie de l'éducation», je rappellerais qu'à ce niveau, en plus d'occulter certains problèmes discutés par des philosophes d'ici et d'ailleurs, je ne vois pas les objectifs de sa recherche. Certes, Allard procède par suggestion, par allusion quant à l'opportunité, à l'actualité de cette conception éducative dans la crise actuelle de notre civilisation. Mais en plus de devoir accepter sans preuve un verdict de notre époque, son propos volontairement abstrait - comme l'exigent les lois du genre "résumé-synthèse» de la pensée de Monsieur X - l'empêche d'établir des connexions avec des données spatio-temporelles. Pourtant, quand Maritain propose de séparer les Hautes Écoles Professionnelles et Techniques de l'Université proprement dite, l'auteur aurait pu expliciter les éléments de ce problème pour Maritain dans le cadre de ceux qui assaillent le milieu universitaire québécois souvent en grève: quels concepts alimentent ces problématiques qui se répètent? quels aspects de la réalité sont explicités dans l'un et l'autre cas? etc.

Mais ce n'était pas là l'entreprise d'Allard. Quoi penser? Quoi cerner? Personnellement, j'ai trop de respect pour notre passé philosophique québécois, pour notre culture d'hier pour laisser croire que Maritain n'a aucune pertinence. Cet homme est venu chez nous, a «influencé» - terme à définir artistes, intellectuels, milieu universitaire, dans le contexte d'une orthodoxie. Cet homme a été au coeur de débats dans Temps Modernes, Esprit, Revue thomiste, La Pensée, revues en conflits, comme le furent et le sont encore les sous-groupes de ma génération qui ont privilégié des thèses opposées. Je soupçonne qu'Allard veut laisser croire que la pédagogie de Maritain mériterait une très grande diffusion à cause de sa Vérité. C'est là une thèse. Je soupçonne Allard de croire que toute saine pédagogie doit s'inspirer de la philosophie thomiste, toujours "vivante», parce qu'elle sait reconnaître des intuitions vraies dans les philosophies antagonistes et parce qu'elle résout des problèmes de notre époque. C'est là une deuxième thèse à propos de la Philosophia perennis. Mais alors, est-ce que ces deux thèses me conduisent à cerner l'enjeu réel mais implicite de l'entreprise d'Allard? J'en ai bien peur. J'ai du respect pour mes collègues de philosophie et une conscience sismique des enjeux théoriques et pratiques qui nous divisent. Surtout en philosophie de l'éducation. En conséquence: je ne puis que signaler sur la base des questions et des problèmes posés dans ce compte rendu que Maritain peut donner lieu à des thèses très différentes de celles-ci... Ce faisant, je souligne au moins d'un trait noir une divergence profonde... pour qu'un jour nous nous trouvions une légitimité qui découle d'une commande sociale.

André Vidricaire UQAM 